\title{
Multiple Gas-Phase Conformations of a Synthetic Linear Poly(acrylamide) Polymer Observed Using Ion Mobility-Mass Spectrometry
}

\author{
Jean R. N. Haler, ${ }^{1}$ Johann Far, ${ }^{1}$ Abdelhafid Aqil, ${ }^{2}$ Jan Claereboudt, ${ }^{3}$ Nick Tomczyk, ${ }^{4}$ \\ Kevin Giles, ${ }^{4}$ Christine Jérôme, ${ }^{2}$ Edwin De Pauw ${ }^{1}$ \\ ${ }^{1}$ Mass Spectrometry Laboratory, University of Liège, Quartier Agora, Allée du Six Aout 11, B-4000, Liège, Belgium \\ ${ }^{2}$ Center for Education and Research on Macromolecules, Department of Chemistry, University of Liège, Quartier Agora, Allée du \\ Six Aout 13, B-4000, Liège, Belgium \\ ${ }^{3}$ Waters Corporation, Connexion Business Park, Brusselsesteenweg 500, 1731, Zellik, Belgium \\ ${ }^{4}$ Waters Corporation, Stamford Ave., Wilmslow, SK9 4AX, UK
}

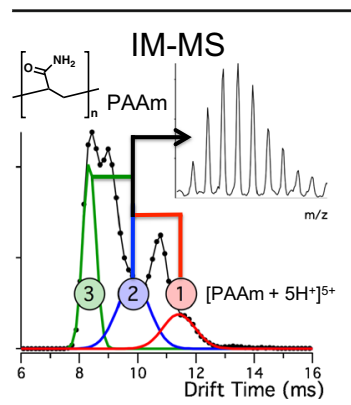

\begin{abstract}
Ion mobility-mass spectrometry (IM-MS) has emerged as a powerful separation and identification tool to characterize synthetic polymer mixtures and topologies (linear, cyclic, star-shaped,....). Electrospray coupled to IM-MS already revealed the coexistence of several charge state-dependent conformations for a single charge state of biomolecules with strong intramolecular interactions, even when limited resolving power IM-MS instruments were used. For synthetic polymers, the sample's polydispersity allows the observation of several chain lengths. A unique collision cross-section (CCS) trend is usually observed when increasing the degree of polymerization (DP) at constant charge state, allowing the deciphering of different polymer topologies. In this paper, we report multiple coexisting CCS trends when increas-
\end{abstract} ing the DP at constant charge state for linear poly(acrylamide) PAAm in the gas phase. This is similar to observations on peptides and proteins. Biomolecules show in addition population changes when collisionally heating the ions. In the case of synthetic PAAm, fragmentation occurred before reaching the energy for conformation conversion. These observations, which were made on two different IM-MS instruments (SYNAPT G2 HDMS and high resolution multi-pass cyclic T-Wave prototype from Waters), limit the use of ion mobility for synthetic polymer topology interpretations to polymers where unique CCS values are observed for each DP at constant charge state.

Keywords: Ion mobility-mass spectrometry, Synthetic polymer, Multiple conformations, Poly(acrylamide), PAAm, Synapt G2, Multi-pass cyclic traveling wave

Received: 30 January 2017/Revised: 14 July 2017/Accepted: 20 July 2017/Published Online: 14 August 2017

\section{Introduction}

Ton mobility spectrometry (IMS) is a gas-phase technique that separates ions according to their mass, their charge, and their shape. During an IMS separation, the ions travel through a gas-filled mobility cell where they undergo collisions with the gas. The extended ions are subjected to more collisions

Electronic supplementary material The online version of this article (doi:10.1007/s13361-017-1769-x) contains supplementary material, which is available to authorized users.

Correspondence to: Jean Haler; e-mail: jean.haler@ulg.ac.be and more drag force than the compact ones, resulting in longer drift times. The collision cross-section (CCS) is determined by the shape of the ions during IMS experiments. The MasonSchamp equation [1] describes the gas-phase mobility $(K)$ of ions submitted to a uniform low electric field and in free molecular regime conditions:

$K=\frac{3}{16} \frac{e}{N}\left[\frac{2 \pi}{\mu k_{B} T}\right]^{\frac{1}{2}} \frac{z}{\Omega}$

where $K$ is the ion mobility, $e$ is the elementary charge, $N$ is the gas number density, $\mu$ is the reduced mass of the ion and the 
drift gas particle, $k_{B}$ is the Boltzmann constant, $T$ is the temperature, $z$ the number of charges of the ion, and $\Omega$ is the CCS.

Ion mobility coupled to mass spectrometry (IM-MS) has emerged a few decades ago as a powerful tool for the analysis of complex polymer mixtures, polymer topologies, and polymer stereoregularity [2-11]. The evolutions of the CCS for the different polymers were monitored as a function of the degree of polymerization (DP) and for different charge states. They could directly be observed from the arrival time distributions (ATDs) of the mass-dispersed polymer samples. Ion activation techniques like collision induced dissociation (CID) and electron capture dissociation (ECD) induce fragmentations of the polymer ions. Fragmentation spectra can be very useful if differences in fragmentation patterns are sufficiently significant between different topologies or stereoregularities of the same polymer [12]. In all cases, IMS brings an additional separation step, simplifying the analysis of crowded MS and MS/MS spectra of polymer mixtures [11-14]. IM-MS separations have thus brought a considerable leap towards polymer mixture analysis.

The use of IMS for polymer mixture and polymer topology characterizations assumes that single, unique drift time values are attributed to each charge state of a polymer ion. This is generally the case for polymers except for biopolymers (e.g., peptides, proteins) for which different stable conformers may coexist in the gas phase for identical residue connectivities [1521]. In this work, we analyzed the IM-MS gas-phase behavior of linear poly(acrylamide). Most of the CCS trends are comparable to those of other synthetic homopolymers [5-8, 2225]. However, several coexisting conformations were observed for small poly(acrylamide) (PAAm) chains bearing five charges. The paper discusses the implications of the coexistence of multiple conformers for the use of IM-MS in the determination of polymer topologies and stereoregularities. In such cases, the polymer topology cannot be straightforwardly inferred from IM-MS data.

\section{Experimental}

\section{Polymer Synthesis}

PAAm was synthesized without stereoselectivity using a controlled radical polymerization, namely the reversible additionfragmentation chain transfer polymerization (RAFT). The synthesis was inspired by Skey and O'Reilly [26], using 2(dodecylthiocarbonothioylthio)-2-methylpropionic acid as RAFT agent. 2,2'-Azobis(2-methylpropionitrile) (AIBN) was used as radical initiator. A detailed description of the atactic polymer synthesis is found in the Supplementary Information.

\section{Ion Mobility-Mass Spectrometry}

PAAm (Figure 1) was solubilized in a mixture $\mathrm{H}_{2} \mathrm{O}$ /methanol (50/50) spiked with $1 \%$ formic acid in order to yield a concentration of polymer ions on the order of $10^{-5} \mathrm{M}$.

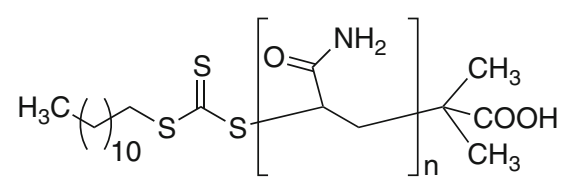

Figure 1. Linear poly(acrylamide) PAAm was synthesized using a reversible addition-fragmentation chain transfer polymerization (RAFT)

The sample was infused into a SYNAPT G2 HDMS traveling wave (T-Wave) ion mobility-mass spectrometer (Waters, Manchester, UK) and into a multi-pass cyclic T-Wave ion mobility-mass spectrometer prototype from Waters [27] with higher resolving power. The resolving power $(\mathrm{CCS} / \triangle \mathrm{CCS})$ of the SYNAPT G2 HDMS setup [28] is around 40, whereas the resolving power of the multi-pass cyclic T-Wave prototype is around 70 when using one cycle (1-pass measurement) and around 150 (estimated on 1+ ions) when using three cycles (3pass measurement). The measurements on the multi-pass cyclic T-Wave were taken using both 1-pass and 3-pass cycles. A detailed description of the IM-MS parameters is found in the Supplementary Information.

The 2D plot of the drift time (SYNAPT G2 HDMS) as a function of the $\mathrm{m} / \mathrm{z}$ of PAAm ions is found in the Supplementary Information (Figure SI3). The processing of the 2D data (IMS and MS) was performed using Waters MassLynx 4.1 software. Data acquisitions on the multi-pass cyclic T-Wave ion mobility-mass spectrometer were performed using Waters' bespoke, in-house software. Data were analyzed using MassLynx 4.1 and Driftscope. ATD peaks were fitted using PeakFit v.4.11 to determine accurate drift times. Data processing was performed using Excel 2011, SigmaPlot 12.0, and IgorPro 6.34A.

\section{Ion Mobility and Arrival Time Distribution processing}

The ATDs obtained from the MassLynx software were imported into the PeakFit v.4.11 software. They were then fitted using Gaussian functions. The appropriate number of Gaussian functions was added until the sum of all fitted Gaussian peaks provided the best coefficient of determination. These Gaussian peaks were then used to illustrate the deconvoluted peaks in the figures of this manuscript. The apex of the fitted Gaussian peaks associated with the different polymer ions provided the precise drift time values and were used for CCS calculations.

\section{Collision Cross-Section Calibration}

The IMS calibration protocol (drift time conversion into collision cross-sections) of Ruotolo and coworkers was followed [29], using proteins and peptides as calibrants (bradykinin [30], tryptic digest of bovine serum albumin (BSA) [31], myoglobin [32], cytochrome $c$ [33], and lysozyme [34]; see Figure SI1 and Table SI1). 


\section{Results and Discussions}

As MS analyses are performed on charged species, the results are obtained as mass-to-charge ratios $(\mathrm{m} / \mathrm{z})$. The $\mathrm{m} / \mathrm{z}$ axis from the mass spectra of the polymer ions can be converted into DP values associated with each of the different charge states $(z)$. Data were consequently plotted in terms of the CCS values as a function of the DPs. Figure 2a represents the plot of the protonated PAAm ions (from $2+$ to $5+$ charge states ranging from DP 10 to DP 130). The CCS evolutions of the $3+$ and the $4+$ charge states show an increase in CCS values at smaller DPs compared with the $2+\mathrm{CCS}$ evolution. The increased Coulomb repulsion at smaller DP values compared with higher DP values for a given charge state $(3+$ or $4+)$ induces a structural rearrangement (different folding) of the protonated polymer ions $[5,6,22-25]$. The polymer chain is thus able to minimize the electrostatic repulsion. At higher DP values for higher charge states $(>2+)$, the CCS evolution merges with the trend given by the $2+$ charge state ions $\left[\mathrm{PAAm}+2 \mathrm{H}^{+}\right]^{2+}$ (black markers in Figure 2a), which is the common trend line. We define the common trend line as the merging CCS tendencies of different charge state polymer ions after having undergone their structural rearrangements to attain maximum intramolecular charge solvation. This common trend line represents the polymer chains being capable of fully stabilizing the carried charge. Such ions are assumed in literature to be spherically shaped $[5,24,25]$ in the gas phase because of the entropic factors constraining the $3 \mathrm{D}$ - arrangement.

Figure $2 \mathrm{~b}$ exhibits an extract of Figure 2a, focusing on the $\left[\mathrm{PAAm}+5 \mathrm{H}^{+}\right]^{5+}$ ions' CCS evolution plotted as a function of the degree of polymerization. For the small DP values of the 5+ ions, three concomitant trends without any structural rearrangements are observed, contrary to the $3+$ and $4+$ ions. Instead, three distinct CCS evolutions are distinguishable. One of these is the evolution of CCS values, which are shifted to around 150-200 $\AA^{2}$ larger values than the CCS evolution of the most compact polymer ions. The trend of these most compact polymer ions is also merged with the common trend line, assuring the most efficient charge solvation. Lastly, an intermediary CCS evolution is median to the two other CCS evolutions. The three simultaneously observed conformations are also distinctly separated as drift time values (non-CCS-converted raw IM-MS data; see Figure 3). The three drift time evolutions are each separated by at least $1 \mathrm{~ms}$ (more than $10 \%$ drift time difference). Figure 3 also exhibits the reproducibility of the three conformations of PAAm using another IM-MS instrument, namely a prototype multi-pass cyclic T-Wave IM-MS instrument (1-pass measurement) from Waters [27]. To build this Figure 3, the experimental IMS conditions of the 1-pass measurements were optimized in order to be in close correlation with the SYNAPT G2 HDMS arrival times. The three drift time evolutions are as well separated by at least $1 \mathrm{~ms}$.

Figure 4 represents the mass spectrum monoisotopicallyextracted ATDs of several $\left[\mathrm{PAAm}+5 \mathrm{H}^{+}\right]^{5+}$ ions at different $\mathrm{DP}$ values $(\mathrm{DP}=56,58,60,62,64,66,68,70,72,74,78)$ originating from measurements taken on the SYNAPT G2 HDMS and on the multi-pass cyclic T-Wave instrument. Figure 4a represents the SYNAPT G2 ATDs and Figure $4 \mathrm{~b}$ and $\mathrm{c}$ represent the 1-pass and 3-pass measurements taken on the multi-pass cyclic T-Wave prototype instrument. The ATD peaks that were not fitted with Gaussian functions correspond to other ions overlapping with the mass spectrum of the [PAAm +
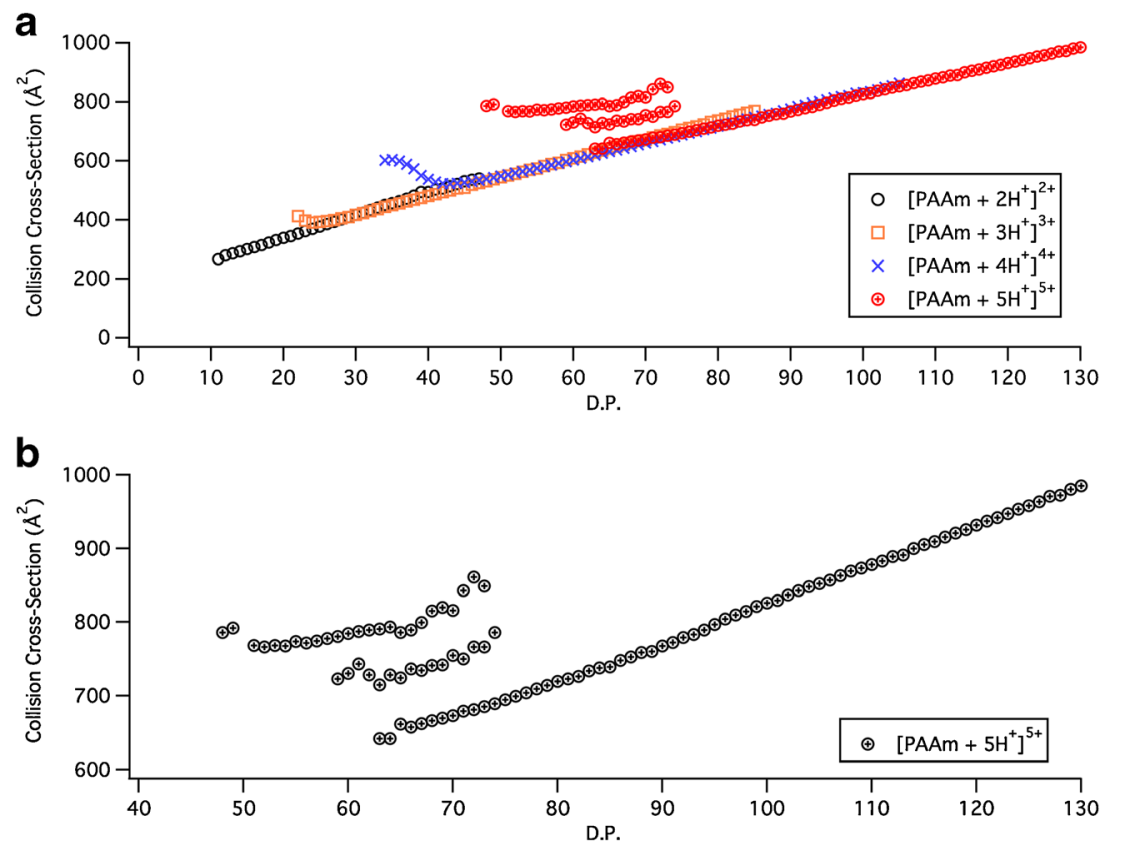

Figure 2. (a) Plot of the measured SYNAPT G2 HDMS T-Wave derived collision cross-section (CCS) versus the DP (degree of polymerization) of linear poly(acrylamide) (PAAm) polymers with varying numbers of protons used to reach different charge states (from $2+$ to $5+$ ). (b) Plot of the CCS evolution as a function of the DP of linear PAAm for the $5+$ charge state ions $\left[\mathrm{PAAm}+5 \mathrm{H}^{+}\right]^{5+}$ 


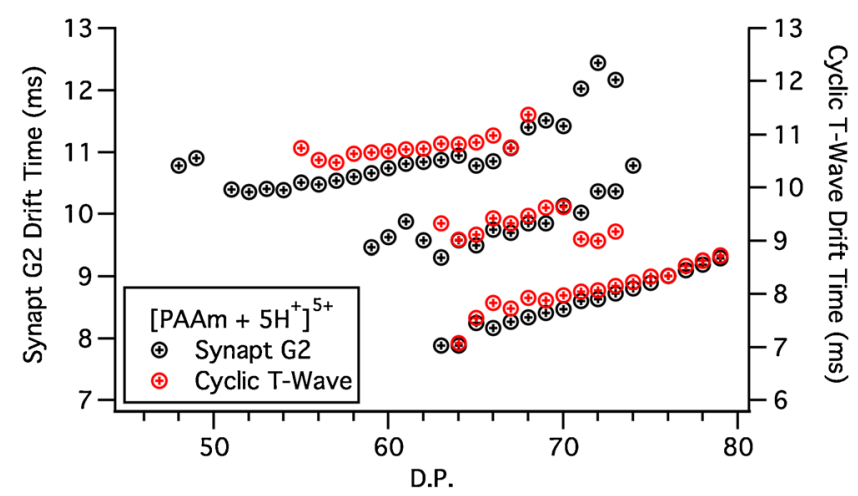

Figure 3. Measured drift times plotted as a function of the DP (degree of polymerization) of linear poly(acrylamide) (PAAm) polymers bearing 5 protons $\left[\mathrm{PAAm}+5 \mathrm{H}^{+}\right]^{5+}$. An extract of the $\mathrm{DP}$ range containing the three coexisting conformations is shown. The black markers represent data measured on the SYNAPT G2 HDMS instrument (left axis); the red markers represent 1-pass data measured on the prototype multi-pass cyclic T-Wave instrument from Waters (right axis). The experimental IMS conditions of this 1-pass measurement were optimized in order to be in close correlation with the SYNAPT G2 HDMS arrival times

$\left.5 \mathrm{H}^{+}\right]^{5+} \mathrm{m} / \mathrm{z}$ peaks (see Figure $5 \mathrm{~b}$ ). As shown in Figure $4 \mathrm{a}$, the more extended conformation 'conformation 1' (highest CCS or drift time values in Figure 2 or Figure 3) is the only observed conformation at smallest DP values $\left(\left[\mathrm{PAAm}_{56}+5 \mathrm{H}^{+}\right]^{5+}\right.$ in Figure 4). Then, the intensity of the second conformation "conformation 2' rises while conformation 1 still is the most abundant conformation ( $\mathrm{DP}=60,62,64)$. Around $\mathrm{DP}=64$, the third and most compact conformation 'conformation 3' (coinciding with the common trend line) appears and becomes rapidly the most abundant conformation (DP > 66). The intensity of conformation 2 does not surpass its relative intensity gained until then $(\mathrm{DP}=66)$. The extended conformation 1's relative intensity has drastically decreased with the appearance of the compact conformation 3 until it finally dies out at higher DP values (DP = $68,72)$, together with conformation 2. At last, $\left[\mathrm{PAAm}_{78}+\right.$ $\left.5 \mathrm{H}^{+}\right]^{5+}(\mathrm{DP}=78)$ exhibits only conformation 3 , the most compact conformation (common trend line). At this stage of the work, the SYNAPT G2 HDMS did not provide satisfactory IMS resolving power (CCS/ $\triangle \mathrm{CCS} \sim 40$ ) to clearly confirm and explain the presence of the observed conformers. We decided to reproduce the experiment using an IMS instrument having higher resolving power. The prototype multi-pass cyclic TWave IMS-ToF was selected and exhibits a drastic improvement of the ion mobility resolving power dependent on the number of passes performed by the instrument $(\mathrm{CCS} / \triangle \mathrm{CCS}$ $\sim 70$ using 1-pass and CCS/ $\triangle \mathrm{CCS} \sim 150$ using 3-pass measurements) coupled with an improved oToF mass analyzer $(65,000$ resolution) [27]. The improved oToF resolution allows for cleaner ATD extractions from the mass spectrum compared with the SYNAPT G2 HDMS. Figure 4b exhibits similar ATD evolutions from the 1-pass measurement on the prototype multi-pass cyclic T-Wave IM-MS (the experimental conditions were optimized for multiple pass measurements; see Supplementary Information). Figure $4 c$, with betterresolved ATDs from the 3-pass measurement, shows as well the three conformations of PAAm. They differ only in relative peak intensities from Figure $4 a$ and $b$. One should note that even with increased ion mobility resolving power, the ATDs of conformation 2 seem surprisingly large, from DP 66 to 70 .

Figure 5a represents the mass spectra extracted from the ATDs (SYNAPT G2 HDMS) of $\left[\mathrm{PAAm}_{68}+5 \mathrm{H}^{+}\right]^{5+}(\mathrm{DP}=68)$. A simulated isotope pattern $\mathrm{m} / \mathrm{z}$ distribution is depicted as well for comparative purposes. Figure $5 \mathrm{~b}$ represents the other ions causing the remaining peaks in the ATD, not fitted with Gaussian functions in Figure 4 and Figure 5a, due to their $\mathrm{m} / \mathrm{z}$ overlaps in the mass spectrum with $\left[\mathrm{PAAm}_{68}+5 \mathrm{H}^{+}\right]^{5+}$.

The mass spectra are extracted at or near the maximum ATD intensities for each of the three conformations (i.e., $11.61,10.09$, and $8.57 \mathrm{~ms})$. As can be noted, the isotope patterns are nearly identical and all three correspond very well to the simulated pattern of $\left[\mathrm{PAAm}_{68}+5 \mathrm{H}^{+}\right]^{5+}$. Each of the MS signals has a high relative abundance, thus indicating the coexistence of the three conformations.

The coexistence of different conformations for one identical polymer could lead to mass spectra misinterpretations during an early-stage IM-MS analysis of polymer topologies and of stereoregular polymer mixtures. Indeed, until now, synthetic (homo)polymers having the same polymer topology were observed to only yield one CCS evolution per charge state [5-8, 22-25]. Hence, if different CCS evolutions are observed for a given charge state during a common mass range (DP range), it could be concluded to be on different polymer topologies or stereoregularities [5-8] present in the mixture. However, linear poly(acrylamide) shows that strong intramolecular interactions in synthetic polymers can lead to multiple trapped conformations in the gas phase. Thus, interpretations of only a small DP range and a (randomly) chosen charge state could lead to false identifications of multiple polymer topologies (or stereoregularities). For monomers that have already been studied in literature, the risk seems decreased when staying in the literature-interpreted charge states. Nevertheless, different polymer chain ends could as well lead to multiple conformations of polymer ions in the gas phase. Additionally, the development of new, higher resolution IM-MS setups [27, 35, 36] could result in multiple conformation interpretations, the separation of which was technically impossible at lower resolving powers.

A potential explanation of the appearance of multiple conformations of linear poly(acrylamide) could be found in poly(peptides). Indeed, poly(acrylamide)s are poly(amide)-based polymers like peptides. Poly(alanine) and poly(glycine), for example, show strong intramolecular interactions resulting in multiple conformations for different charge states [15-18]. Multiple PAAm conformations can hence be compared through their amide functions to peptides. Different hydrogen bonding combinations between PAAm monomer units could result in stable coexisting conformations yielding minimized Coulomb repulsions and leading to different CCS evolutions as a function of the mass (or the DP). 

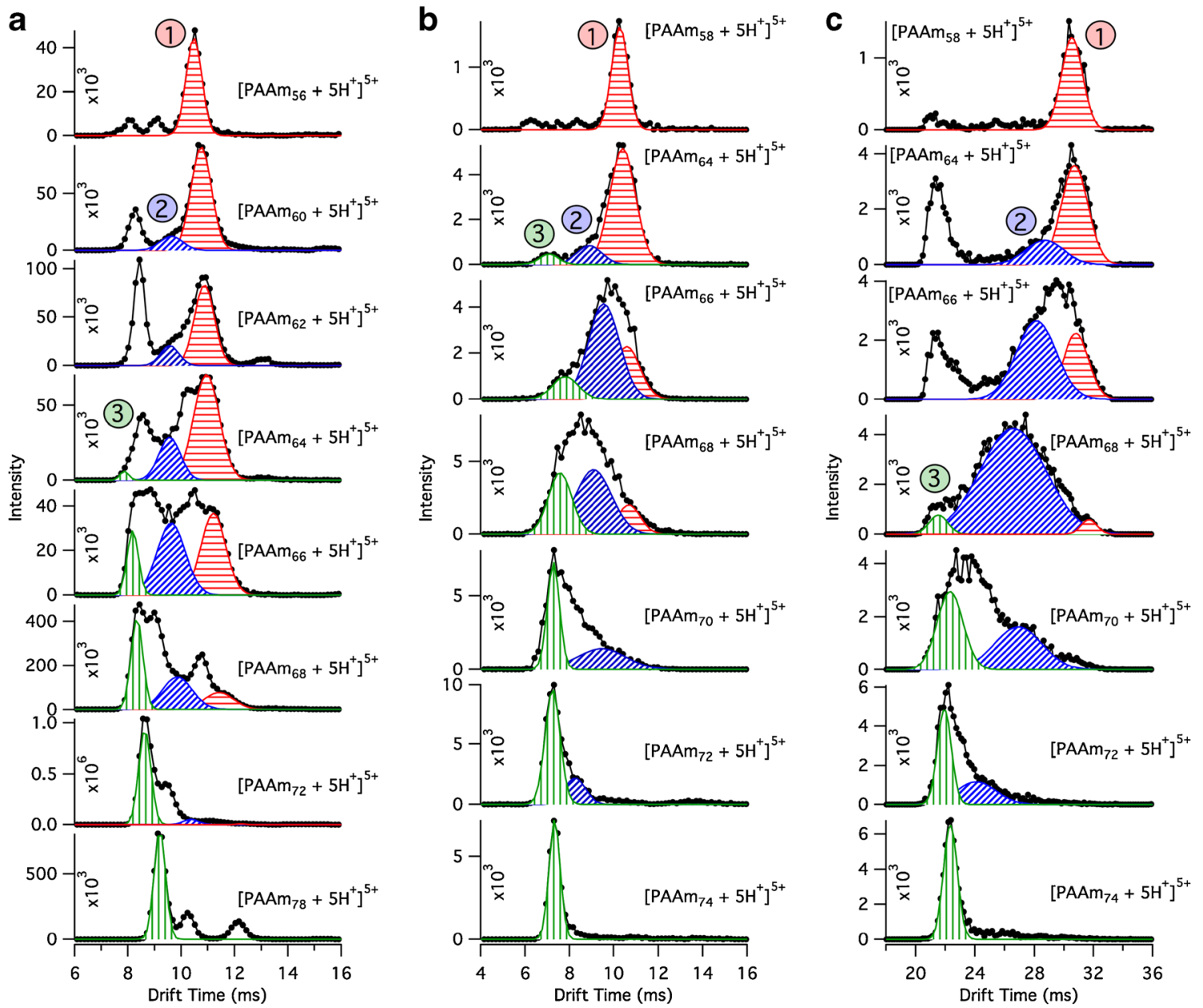

d

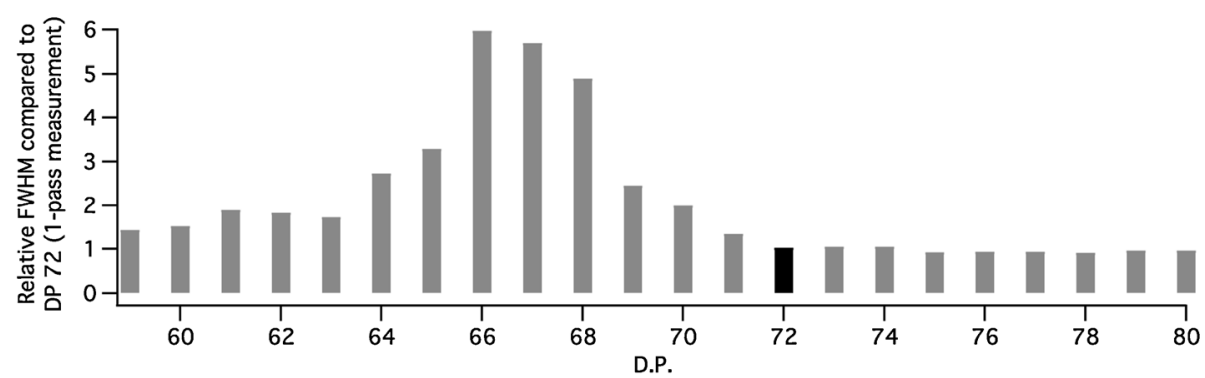

Figure 4. Monoisotopically-extracted arrival time distributions (ATDs) from the mass spectrum of different $\left[\mathrm{PAAm} \mathrm{DP}^{+} 5 \mathrm{H}^{+}\right]^{5+}$ ions $(\mathrm{DP}=56,58,60,62,64,66,68,70,72,74,78)$ (black markers). The red (conformation 1; horizontally-striped pattern), blue (conformation 2; diagonally-striped pattern), and green (conformation 3; vertically-striped pattern) lines represent the ATD fits (Gaussian peaks) performed to extract precise drift time values (used in Figure 3 and for drift time-CCS conversions in Figure 2). The peaks not fitted with Gaussian functions correspond to other ions overlapping in the mass spectrum with the $\left[\mathrm{PAAm} \mathrm{DP}_{\mathrm{DP}}+5 \mathrm{H}^{+}\right]^{5+}$ $\mathrm{m} / \mathrm{z}$ peaks (see Figure 5b). (a) Represents the ATDs measured on the SYNAPT G2 HDMS instrument (CCS/ $\triangle \mathrm{CCS} \sim 40$ [28]). (b) Represents data analyzed from 1-pass measurements on the multi-pass cyclic T-Wave prototype (CCS/ $\triangle \mathrm{CCS} \sim 70$; the experimental conditions are reported in the Supplementary Information), and (c) exhibits data from 3-pass measurements (CCS/ $\triangle$ CCS $\sim 150$ as estimated on $1+$ ions) [27]. (d) Provides the evolution of the relative peak width at half height (normalized towards DP $=72$ ) of PAAm ions during 1-pass IMS measurements

In addition, the observed multiple populations of poly(peptides) are temperature- dependent and can be shifted from one preferable conformation to the other as a function of ion heating. The multiple observed conformations of PAAm in the gas phase might hence be related to the existence of conformations, trapped during the ESI process, for which the conversion energy barrier would be too high to overcome. However, unlike poly(peptides), such as poly(alanine) or poly(glycine), the three populations of PAAm do not shift towards one preferential conformation upon ion heating 

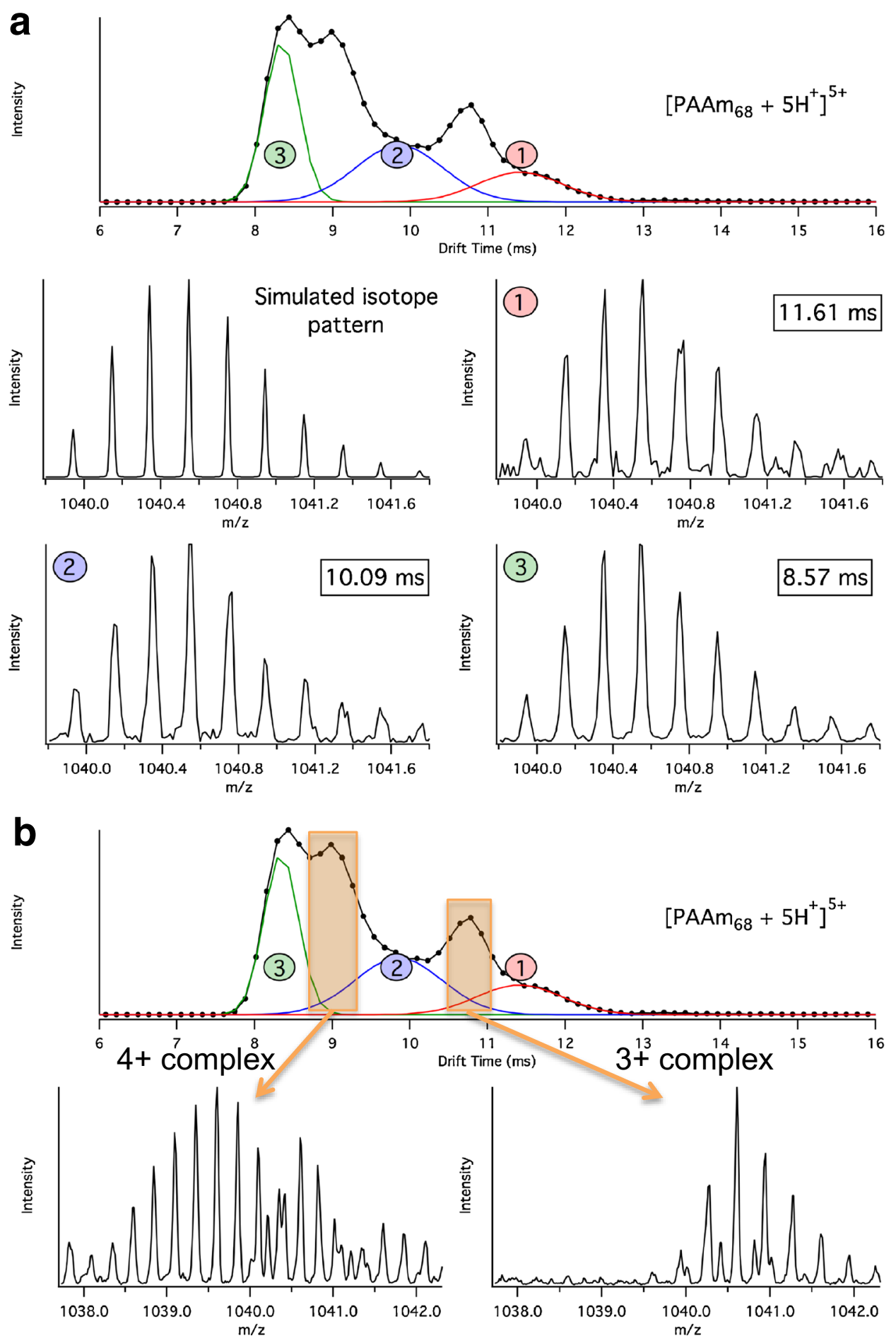

Figure 5. Monoisotopically - extracted arrival time distribution (ATD; SYNAPT G2 HDMS) of the $\left[P A A m_{68}+5 \mathrm{H}^{+}\right]^{5+}$ ion $(\mathrm{DP}=68$ ) and the corresponding mass spectra extracted at different drift times. (a) The red, blue, and green Gaussian peaks represent the ATD fits performed to extract precise drift time values (used in Figure 3 and for drift time-CCS conversions in Figure 2). The first observed extended conformation 'conformation 1' at higher drift times is depicted in red, the second, intermediate conformation 'conformation 2 ' is depicted in blue, and the most compact conformation 'conformation 3 ' is depicted in green. The drift time-extracted mass spectra for the respective conformations are identically numbered and color-coded. They are identified as well through the drift time annotation. The $\left[\mathrm{PAAm}_{68}+5 \mathrm{H}^{+}\right]^{5+}$ mass spectra were extracted at or near the maximum ATD intensity: at $11.61 \mathrm{~ms}$ for conformation 1 , at $10.09 \mathrm{~ms}$ for conformation 2 , and at $8.57 \mathrm{~ms}$ for conformation 3 . A comparative simulated $\mathrm{m} / \mathrm{z}$ isotope pattern is shown as well. (b) The peaks not fitted with Gaussian functions, framed by the orange boxes, correspond to other ions overlapping in the mass spectrum with the $\left[\mathrm{PAAm}_{68}+5 \mathrm{H}^{+}\right]^{5+} \mathrm{m} / \mathrm{z}$ isotope distribution. The $\mathrm{m} / \mathrm{z}$ overlap at smaller drift times (around $9 \mathrm{~ms}$ ) corresponds to a $4+$ charged ion, and the $\mathrm{m} / \mathrm{z}$ overlapping ion at higher drift times corresponds to a $3+$ charged polymer ion 
$\left(\left[\mathrm{PAAm}_{68}+5 \mathrm{H}^{+}\right]^{5+}\right.$, Figure SI2) on the SYNAPT G2 HDMS. When increasing the accelerating voltage (trap DC bias, collisional induced ion heating) from 45 to $75 \mathrm{~V}$ (Figure SI2), all the conformations seem to undergo the same signal decrease until favoring ion fragmentation at $90 \mathrm{~V}$. The fragmentation pathway thus seems more favorable than the conversion pathway when heating the ions after creating the initial conformation population during the ESI process. The observed preferential fragmentation upon increased ion heating could as well be at the origin of the ATD signal intensity differences between the multi-pass cyclic T-Wave measurements and the SYNAPT G2 HDMS (see Figure 4). One could contemplate analyzing PAAm complexes on IMS-IMS setups [37, 38] inducing less ion heating during the ESI process in order to be potentially able to observe different population ratios of the system [39].

Improving the ion mobility resolving power was expected to provide better resolved ATD peaks of the observed DPs of PAAm, especially around the value of DP 68. Interestingly, we instead observed the broadening of the ATD when we approached DP 68 (as shown in Figure 4d). Here we provide an attempt to explain the experimental results on the basis of higher ion mobility resolving power (3-pass measurements).

A first explanation of the broadening of the IMS peak of conformation 2 could be found in the possible existence of an equilibrium between conformation 1 and conformation 3 [39] for which conformation 2 could be a transitory state. However, the ion heating experiments do not suggest any major population shifts between the conformations, nor the disappearance of denoted conformation 2 before the two other conformations.

A different explanation of the IMS peak broadening of conformation 2 could then be established. Let us assume the following definition of floppy structures or 'floppiness': all metastable conformations form a continuum of conformations separated by energy barriers smaller than $k T$, and not exceeding the barriers of non-exchanging (i.e., stable) conformations. In the case of PAAm from DP 66 to 68, it appears that the ATD peak broadening, despite the improved resolving power of the IMS apparatus, can be reasonably explained by the presence of metastable conformers of the polymer ions. These metastable conformers all share close CCS values. This happens notably through the absence of interactions between the additional monomers during the polymer chain elongation (from DP 66 to 68 ). These additional monomers have more freedom in terms of motion with regard to the core of the polymer chain and introduce a floppy section in the polymer structure. This floppy part contributes to the peak broadening because of a continuum of dynamic structures having close ATDs. After sufficient chain elongation, the sum of the individual monomer interactions and the entropic contribution will again allow for polymer chain compaction and the loss of the floppy section. These hypotheses have to be confirmed by further work. We eventually can hypothesize about the global shape and the floppy part of the polymer as an apparently spherical shape (as seen by the IMS gas) having one, two, or even more growing excrescence(s). In this case, IMS alone should not be able to provide strong evidence concerning the polymer ions' shape, even if much higher resolving power is used, because of the intrinsic dynamics of this system. IMS hyphenated with e.g., spectroscopic studies should provide improved data for 3D structure elucidation.

\section{Conclusion}

Usually, synthetic polymer IMS analyses yield unique CCS values for each DP at constant charge state[5-8, 22-25]. We observed three coexisting conformations for linear synthetic poly(acrylamide) $\left[\mathrm{PAAm}+5 \mathrm{H}^{+}\right]^{5+}$ ions. The three conformations evolve separately during a range of around 20 degrees of polymerization (from DP 48 to DP 74). The drift time peaks of the three conformations are observed with a drift time difference of around (or at least) $1 \mathrm{~ms}$ (i.e., CCS separation $\sim 70-100$ $\AA^{2}$ ) on a commercially available SYNAPT G2 HDMS setup.

Multiple conformations are commonly observed in literature for peptides [15-18] due to differing hydrogen bond combinations. Such differing hydrogen bond combinations could as well explain the multiple conformations of poly(acrylamide). However, contrary to biomolecules such as peptides, conformer conversions do not seem to be the preferable pathway upon ion heating of PAAm. The polymer chain fragmentation pathway was favored.

Owing to high resolution IMS data obtained on a prototype multi-pass cyclic T-Wave [27], the intermediate IMS peak was shown to broaden during a small DP range. This observation was discussed as a set of structures having only small structural differences resulting in close CCS values, due to small energy barriers separating the numerous conformations.

Given the reproducibility of the multiple conformations of PAAm on two different IM-MS setups (SYNAPT G2 HDMS and multi-pass cyclic T-Wave prototype from Waters [27]), this points to a more general conclusion. Conclusions on topology and stereoregularity of synthetic polymers [5-8] are based on the assumption of unique CCS values associated with each DP at constant charge state. We observed three coexisting conformations for a single polymer topology. This prevents the deciphering of topologies of such synthetic polymers in the affected DP range. In order to interpret complex polymer mixtures, the assumption of unique CCS values for each polymer ion has to be validated. Polymer standards and their potentially involved topologies should hence be first analyzed separately. Interpreting only small DP ranges for (randomly) chosen charge states should be avoided, considering misinterpretation of data in terms of potential topologies (or stereoregularities).

\section{Acknowledgements}

The authors acknowledge financial support of the F.R.S.FNRS (J.R.N.H. is a FRIA Doctorate Fellow). Philippe Massonnet (FRIA Doctorate Fellow, F.R.S.-FNRS) is thanked for his helpful discussions on this paper. Justine Parmentier is acknowledged for technical assistance. Waters Corporation is thanked for providing early access to their multi-pass cyclic TWave prototype. 


\section{Compliance with Ethical Standards}

\author{
Conflict of Interest The authors declare no competing finan- \\ cial interest.
}

\section{References}

1. Mason, E.A., McDaniel, E.W.: Transport properties of ions in gases. Wiley, New York (1988)

2. Gidden, J., Wyttenbach, T., Jackson, A.T., Scrivens, J.H., Bowers, M.T.: Gas-Phase conformations of synthetic polymers: poly(ethylene glycol), poly(propylene glycol), and poly(tetramethylene glycol). J. Am. Chem. Soc. 122, 4692-4699 (2000)

3. Bagal, D., Zhang, H., Schnier, P.D.: Gas-phase proton-transfer chemistry coupled with TOF mass spectrometry and ion mobility-MS for the facile analysis of poly(ethylene glycols) and PEGylated polypeptide conjugates. Anal. Chem. 80, 2408-2418 (2008)

4. Trimpin, S., Clemmer, D.E.: Ion mobility spectrometry/mass spectrometry snapshots for assessing the molecular compositions of complex polymeric systems. Anal. Chem. 80, 9073-9083 (2008)

5. Morsa, D., Defize, T., Dehareng, D., Jérôme, C., De Pauw, E.: Polymer topology revealed by ion mobility coupled with mass spectrometry. Anal. Chem. 86, 9693-9700 (2014)

6. Hoskins, J.N., Trimpin, S., Grayson, S.M.: Architectural differentiation of linear and cyclic polymeric isomers by ion mobility spectrometry-mass spectrometry. Macromolecules 44, 6915-6918 (2011)

7. Foley, C.D., Zhang, B., Alb, A.M., Trimpin, S., Grayson, S.M.: Use of ion mobility spectrometry-mass spectrometry to elucidate architectural dispersity within star polymers. ACS Macro Lett. 4, 778-782 (2015)

8. Kim, K., Lee, J.W., Chang, T., Kim, H.I.: Characterization of polylactides with different stereoregularity using electrospray ionization ion mobility mass spectrometry. J. Am. Soc. Mass Spectrom. 25, 1771-1779 (2014)

9. Fischer, J.L., Lutomski, C.A., El-Baba, T.J., Siriwardena-Mahanama, B.N., Weidner, S.M., Falkenhagen, J., Allen, M.J., Trimpin, S.: Matrixassisted ionization-ion mobility spectrometry-mass spectrometry: selective analysis of a europium-PEG complex in a crude mixture. J. Am. Soc. Mass Spectrom. 26, 2086-2095 (2015)

10. Barrère, C., Selmi, W., Hubert-Roux, M., Coupin, T., Assumani, B., Afonso, C., Giusti, P.: Rapid analysis of polyester and polyethylene blends by ion mobility-mass spectrometry. Polym. Chem. 5, 3576-3582 (2014)

11. Crotty, S., Gerişlioğlu, S., Endres, K.J., Wesdemiotis, C., Schubert, U.S.: Polymer architectures via mass spectrometry and hyphenated techniques: A review. Anal. Chim. Acta 932, 1-21 (2016)

12. Josse, T., De Winter, J., Dubois, P., Coulembier, O., Gerbaux, P., Memboeuf, A.: A tandem mass spectrometry-based method to assess the architectural purity of synthetic polymers: a case of a cyclic polylactide obtained by click chemistry. Polym. Chem. 6, 64-69 (2015)

13. Kaczorowska, M.A., Cooper, H.J.: Electron capture dissociation, electron detachment dissociation, and collision-induced dissociation of polyamidoamine (PAMAM) dendrimer ions with amino, amidoethanol, and sodium carboxylate surface groups. J. Am. Soc. Mass Spectrom. 19, 1312-1319 (2008)

14. Hilton, G.R., Jackson, A.T., Thalassinos, K., Scrivens, J.H.: Structural analysis of synthetic polymer mixtures using ion mobility and tandem mass spectrometry. Anal. Chem. 80, 9720-9725 (2008)

15. Counterman, A.E., Clemmer, D.E.: Gas phase polyalanine: assessment of $\mathrm{i} \rightarrow \mathrm{i}+3$, and $\mathrm{i} \rightarrow \mathrm{i}+4$ helical turns in [Alan $+4 \mathrm{H}] 4+(\mathrm{n}=29-49)$ ion. $\mathrm{J}$. Phys. Chem. B 106, 12045-12051 (2002)

16. Breaux, G.A., Jarrold, M.F.: Probing helix formation in unsolvated peptides. J. Am. Chem. Soc. 125, 10740-10747 (2003)

17. Counterman, A.E., Clemmer, D.E.: Compact $\rightarrow$ extended helix transitions of polyalanine in vacuo. J. Phys. Chem. B 107, 21112117 (2003)

18. Rossi, M., Blum, V., Kupser, P., von Helden, G., Bierau, F., Pagel, K., Meijer, G., Scheffler, M.: Secondary structure of Ac-Ala n -LysH + polyalanine peptides $(\mathrm{n}=5,10,15)$ in vacuo: helical or not? J. Phys. Chem. Lett. 1, 3465-3470 (2010)

19. Chen, S.-H., Russell, D.H.: How closely related are conformations of protein ions sampled by IM-MS to native solution structures? J. Am. Soc. Mass Spectrom. 26, 1433-43 (2015)

20. Valentine, S.J., Counterman, A.E., Clemmer, D.E.: Conformerdependent proton-transfer reactions of ubiquitin ions. J. Am. Soc. Mass Spectrom. 8, 954-961 (1997)

21. Shelimov, K.B., Jarrold, M.F.: Conformations, unfolding, and refolding of apomyoglobin in vacuum: an activation barrier for gas-phase protein folding. J. Am. Chem. Soc. 119, 2987-2994 (1997)

22. Ude, S., de la Mora Fernandez, J., Thomson, B.A.: Charge-induced unfolding of multiply charged polyethylene glycol ions. J. Am. Chem. Soc. 126, 12184-12190 (2004)

23. Trimpin, S., Plasencia, M., Isailovic, D., Clemmer, D.E.: Resolving oligomers from fully grown polymers with IMS-MS. Anal. Chem. 79, 7965-7974 (2007)

24. De Winter, J., Lemaur, V., Ballivian, R., Chirot, F., Coulembier, O., Antoine, R., Lemoine, J., Cornil, J., Dubois, P., Dugourd, P., Gerbaux, P.: Size dependence of the folding of multiply charged sodium cationized polylactides revealed by ion mobility mass spectrometry and molecular modeling. Chem. - A Eur. J. 17, 9738-9745 (2011)

25. Larriba, C.: Fernandez de la Mora, J.: The gas phase structure of Coulombically stretched polyethylene glycol ions. J. Phys. Chem. B 116, 593-598 (2012)

26. Skey, J., O'Reilly, R.K.: Facile one pot synthesis of a range of reversible addition-fragmentation chain transfer (RAFT) agents. Chem. Commun. 4183-4185 (2008)

27. Giles, K., Ujma, J., Wildgoose, J., Green, M., Richardson, K., Langridge, D., Tomczyk, N.: Design and performance of a second-generation cyclic ion mobility enabled Q-ToF, June 6 (Poster Presentation). In: Proceedings of the 65th Conference on Mass Spectrometry and Allied Topics, Indianapolis, IN, June 4-8 (2017)

28. Giles, K., Williams, J.P., Campuzano, I.: Enhancements in traveling wave ion mobility resolution. Rapid Commun. Mass Spectrom. 25, 1559-1566 (2011)

29. Ruotolo, B.T., Benesch, J.L.P., Sandercock, A.M., Hyung, S.-J., Robinson, C.V.: Ion mobility-mass spectrometry analysis of large protein complexes. Nat. Protoc. 3, 1139-1152 (2008)

30. Counterman, A.E., Valentine, S.J., Srebalus, C.A., Henderson, S.C., Hoaglund, C.S., Clemmer, D.E.: High-order structure and dissociation of gaseous peptide aggregates that are hidden in mass spectra. J. Am. Soc. Mass Spectrom. 9, 743-759 (1998)

31. Bush, M.F., Campuzano, I.D.G., Robinson, C.V.: Ion mobility mass spectrometry of peptide ions: effects of drift gas and calibration strategies. Anal. Chem. 84, 7124-7130 (2012)

32. Shelimov, K.B., Jarrold, M.F.: Vacuum : An activation barrier for gasphase protein folding. J. Am. Chem. Soc. 119, 2987-2994 (1997)

33. Chen, Y.L., Collings, B.A., Douglas, D.J.: Collision cross-sections of myoglobin and cytochrome $c$ ions with Ne, Ar, and Kr. J. Am. Soc. Mass Spectrom. 8, 681-687 (1997)

34. Valentine, S.J., Anderson, J.G., Ellington, A.D., Clemmer, D.E.: Disulfide-intact and -reduced lysozyme in the gas phase: conformations and pathways of folding and unfolding. J. Phys. Chem. B 101, 38913900 (1997)

35. Michelmann, K., Silveira, J.A., Ridgeway, M.E., Park, M.A.: Fundamentals of trapped ion mobility spectrometry. J. Am. Soc. Mass Spectrom. 26, 14-24 (2015)

36. Silveira, J.A., Ridgeway, M.E., Park, M.A.: High resolution trapped ion mobility spectrometery of peptides. Anal. Chem. 86, 5624-7 (2014)

37. Kurulugama, R.T., Valentine, S.J., Sowell, R.A., Clemmer, D.E.: Development of a high-throughput IMS-IMS-MS approach for analyzing mixtures of biomolecules. J. Proteom. 71, 318-331 (2008)

38. Simon, A.-L., Chirot, F., Min Choi, C., Clavier, C., Barbaire, M., Maurelli, J., Dagany, X., MacAleese, L., Dugourd, P.: Tandem ion mobility spectrometry coupled to laser excitation. Rev. Sci. Instrum. 86, 94101 (2015)

39. Poyer, S., Comby-Zerbino, C., Choi, C.M., MacAleese, L., Deo, C., Bogliotti, N., Xie, J., Salpin, J.-Y., Dugourd, P., Chirot, F.: Conformational dynamics in ion mobility data. Anal. Chem. 89, 4230-4237 (2017) 